\title{
The Exponentiated Kumaraswamy Inverse Weibull Distribution with Application in Survival Analysis
}

\author{
J. A. Rodrigues \\ Department of Education, Federal Institute of Bahia \\ Feira de Santana, 44.096-486, Brazil \\ jailsondearaujo@yahoo.com.br \\ A. P. C. M. Silva \\ Department of Exact and Biological Sciences, Federal University of São João Del Rei \\ Sete Lagoas, 35701-970, Brazil \\ apcmadeira@hotmail.com \\ G.G. Hamedani \\ Department of Mathematics, Statistics and Computer Science \\ Marquette University \\ Milwaukee, Wisconsin 53201-1881
}

Received 29 January 2015

Accepted 14 July 2015

\begin{abstract}
In this paper, a new distribution called the exponentiated Kumaraswamy inverse Weibull is proposed. This distribution includes as special cases the inverse exponential, inverse Weibull, inverse Rayleigh and exponentiated inverse Weibull distributions. We study the main properties of this distribution, with special emphasis on its moments and some characteristics related to reliability studies. We also discuss parameter estimation considering the methods of moments and maximum likelihood. An application reveals that the model proposed can be very useful in fitting real data.
\end{abstract}

Keywords: Akaike information criterion; hazard function; Kumaraswamy distribution; maximum likelihood estimation; Weibull distribution; characterizations.

\section{Introduction}

The inverse Weibull distribution usually used in reliability and biological studies. For many years, researchers have been developing various extensions and modified forms of the inverse Weibull distribution. Recently, [2] introduced an extension of the inverse Weibull distribution called the beta generalized inverse Weibull distribution. [5] defined a three-parameter generalized inverse Weibull distribution with decreasing and unimodal failure rate. [9] proposed the modified inverse Weibull 


\section{J. A. Rodrigues et al.}

distribution and discussed its various properties. [10] introduced and studied a four-parameter distribution, so-called the beta inverse Weibull distribution and [13] introduced and studied a fourparameter Inverse Weibull distribution.

The cumulative distribution function (CDF) and probability density function (PDF) of the inverse Weibull distribution are given by

$$
G(x ; \alpha, \beta)=\exp \left[-\left(\frac{\alpha}{x}\right)^{\beta}\right]
$$

and

$$
g(x ; \alpha, \beta)=\beta \alpha^{\beta} x^{-(\beta+1)} \exp \left[-\left(\frac{\alpha}{x}\right)^{\beta}\right]
$$

respectively, where $x>0, \alpha>0$ and $\beta>0$.

In this paper, a new distribution called the exponentiated Kumaraswamy inverse Weibull is proposed. This distribution includes as special cases the inverse exponential, inverse Weibull, inverse Rayleigh and exponentiated inverse Weibull distributions. The study examines various properties of the new model. The flexibility of this distribution is illustrated in an application to a real data set.

The rest of the paper is organized as follows. In Section 2 the exponentiated Kumaraswamy inverse Weibull distribution is defined and some special sub-models are discussed. Various structural properties including moments, mean deviations and Rényi entropy of the exponentiated Kumaraswamy inverse Weibull distribution are explored in Section 3. Characterizations of the new model are presented in Section 4. The estimation of the model parameters using the methods of moments and maximum likelihood is discussed in Section 5. Finally, in Section 6 an application on a real data set is reported.

\section{The model}

[11] introduced a two-parameter distribution, known as Kumaraswamy distribution, given by its $\mathrm{CDF}$

$$
F(x ; \lambda, \eta)=1-\left(1-x^{\lambda}\right)^{\eta}
$$

where $0<x<1, \lambda>0$ and $\eta>0$. The corresponding CDF for (2.1) is given by

$$
f(x ; \lambda, \eta)=\lambda \eta x^{\lambda-1}\left(1-x^{\lambda}\right)^{\eta-1}
$$

The Kumaraswamy distribution has been identified as a viable alternative to Beta distribution because they both have the same basic shape properties (unimodal, uniantimodal, increasing, decreaing, monotone or constant). However, the PDF given in Equation 2.2 does not involve any incomplete beta function ratio and it is regarded as being tractable because of its mild algebraic properties. Recently, [12] proposed a generalization of the Kumaraswamy distribution, so-called the exponentiated Kumaraswamy distribution. The CDF and PDF of the exponentiated Kumaraswamy 
distribution are given by

$$
F(x ; \lambda, \eta, \theta)=\left[1-\left(1-x^{\lambda}\right)^{\eta}\right]^{\theta}
$$

and

$$
f(x ; \lambda, \eta, \theta)=\lambda \theta \eta x^{\lambda-1}\left(1-x^{\lambda}\right)^{\eta-1}\left[1-\left(1-x^{\lambda}\right)^{\eta}\right]^{\theta-1}
$$

respectively, where $0<x<1, \lambda>0, \eta>0$ and $\theta>0$.

Let $G(x)$ be the CDF of a random variable $X$. The CDF of a generalized class of distributions is given by

$$
F(x ; \lambda, \eta, \theta)=\left[1-\left(1-G(x)^{\lambda}\right)^{\eta}\right]^{\theta}
$$

The corresponding PDF to (2.5) is

$$
f(x ; \lambda, \eta, \theta)=\lambda \theta \eta g(x) G(x)^{\lambda-1}\left(1-G(x)^{\lambda}\right)^{\eta-1}\left[1-\left(1-G(x)^{\lambda}\right)^{\eta}\right]^{\theta-1}
$$

[7] have used the CDF of Dagum distribution in (2.6) to propose the exponentiated Kumaraswamy-Dagum distribution. The generalization (2.6) can be used to propose other distributions based on the exponentiated Kumaraswamy distribution.

Replacing (1.1) in (2.5) we obtain a new distribution, called exponentiated Kumaraswamy inverse Weibull (EKIW), with CDF given by

$$
F(x ; \alpha, \beta, \lambda, \eta, \theta)=\left[1-\left(1-\exp \left[-\lambda\left(\frac{\alpha}{x}\right)^{\beta}\right]\right)^{\eta}\right]^{\theta}
$$

and PDF

$$
\begin{aligned}
f(x ; \alpha, \beta, \lambda, \eta, \theta) & =\beta \lambda \theta \eta \alpha^{\beta} x^{-(\beta+1)} \exp \left[-\lambda\left(\frac{\alpha}{x}\right)^{\beta}\right]\left(1-\exp \left[-\lambda\left(\frac{\alpha}{x}\right)^{\beta}\right]\right)^{\eta-1} \\
& \times\left[1-\left(1-\exp \left[-\lambda\left(\frac{\alpha}{x}\right)^{\beta}\right]\right)^{\eta}\right]^{\theta-1}
\end{aligned}
$$

Figure 1 shows the different graphs of PDF of EKIW distribution for different values of the parameters $\alpha, \beta, \lambda, \eta$ and $\theta$. 


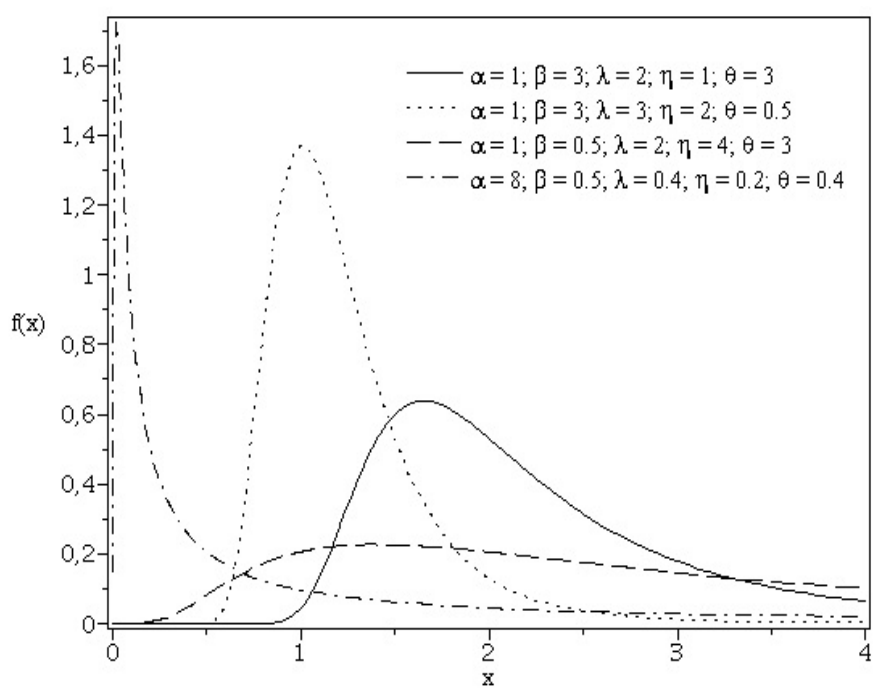

Fig. 1. The PDF of the EKIW distribution for different values of the parameters.

\subsection{Sub-models}

Sub-models of EKIW distribution for selected values of the parameters are presented in this subsection.

(1) When $\beta=\lambda=\eta=\theta=1$, the EKIW distribution is the inverse exponential distribution introduced by [8], with the density given by

$$
f(x ; \alpha)=\alpha x^{-2} \exp \left[-\left(\frac{\alpha}{x}\right)\right] ;
$$

(2) If $\beta=2$ and $\lambda=\eta=\theta=1$, the EKIW distribution is the inverse Rayleigh distribution introduced by [14]. The PDF is given by

$$
f(x ; \alpha)=2 \alpha^{2} x^{-3} \exp \left[-\left(\frac{\alpha}{x}\right)^{2}\right]
$$

(3) If $\lambda=\eta=\theta=1$, we have the inverse Weibull (IW) distribution with the PDF,

$$
f(x ; \alpha, \beta)=\beta \alpha^{\beta} x^{-(\beta+1)} \exp \left[-\left(\frac{\alpha}{x}\right)^{\beta}\right]
$$

(4) If $\eta=\theta=1$, we have the generalized inverse Weibull (GIW) introduced by [5]. The corresponding $\mathrm{PDF}$ is

$$
f(x ; \alpha, \beta, \lambda)=\beta \lambda \alpha^{\beta} x^{-(\beta+1)} \exp \left[-\lambda\left(\frac{\alpha}{x}\right)^{\beta}\right]
$$

(5) When $\lambda=\eta=1$, the EKIW distribution is the GIW distribution with parameters $\alpha, \beta$ and $\theta$. The corresponding PDF is 


$$
f(x ; \alpha, \beta, \theta)=\beta \theta \alpha^{\beta} x^{-(\beta+1)} \exp \left[-\theta\left(\frac{\alpha}{x}\right)^{\beta}\right]
$$

(6) If $\eta=1$, we have another GIW distribution with parameters $\alpha, \beta, \lambda \theta$ and PDF is given by

$$
f(x ; \alpha, \beta, \lambda \theta)=\beta \lambda \theta \alpha^{\beta} x^{-(\beta+1)} \exp \left[-\lambda \theta\left(\frac{\alpha}{x}\right)^{\beta}\right]
$$

\section{Properties of the EKIW model}

\subsection{Expansions for the cumulative and density functions}

For any real non-integer $\theta>0$, we have the power series

$$
(1-\omega)^{\theta-1}=\sum_{j=0}^{\infty} \frac{(-1)^{j} \Gamma(\theta) \omega^{j}}{\Gamma(\theta-j) j !}
$$

where $|\omega|<1$ and $\Gamma(\cdot)$ is the gamma function defined by

$$
\Gamma(\alpha)=\int_{0}^{\infty} t^{\alpha-1} e^{-t} d t
$$

Using the power series (3.1) in Equation (2.7), we can write

$$
F(x ; \alpha, \beta, \lambda, \eta, \theta)=\theta \sum_{j=0}^{\infty} \frac{(-1)^{j} \Gamma(\theta)}{\Gamma(\theta-j+1) j !}\left(1-\exp \left[-\lambda\left(\frac{\alpha}{x}\right)^{\beta}\right]\right)^{j \eta}
$$

For $\theta$ integer, the index $j$ in the previous sums stops at $\theta$. Using again the power series in Equation (3.3), we can express (2.7) (for $\eta>0$ real non-integer) as

$$
F(x ; \alpha, \beta, \lambda, \eta, \theta)=\theta \eta \sum_{j=0}^{\infty} \sum_{k=0}^{\infty} \frac{(-1)^{j+k} \Gamma(\theta) \Gamma(j \eta)}{\Gamma(\theta-j+1) \Gamma(j \eta-k+1)(j-1) ! k !} \exp \left[-\lambda k\left(\frac{\alpha}{x}\right)^{\beta}\right]
$$

Now, using the power series (3.1) in the Equation (2.8), we obtain

$$
f(x ; \alpha, \beta, \lambda, \eta, \theta)=\frac{\beta \lambda \theta \eta \alpha^{\beta}}{x^{\beta+1}} \sum_{j=0}^{\infty} \sum_{k=0}^{\infty} \frac{(-1)^{j+k} \Gamma(\theta) \Gamma[\eta(j+1)]}{\Gamma(\theta-j) \Gamma[\eta(j+1)-k] j ! k !} \exp \left[-\lambda(k+1)\left(\frac{\alpha}{x}\right)^{\beta}\right]
$$




\section{J. A. Rodrigues et al.}

\subsection{Hazard and reverse hazard functions}

For a continuous distribution with $\operatorname{PDF} f(x)$ and $\operatorname{CDF} F(x)$, the hazard function is defined as

$$
h(x)=\lim _{\Delta x \rightarrow 0} \frac{P(X<x+\Delta x \mid X>x)}{\Delta x}=\frac{f(x)}{1-F(x)}
$$

The hazard function is an important quantity characterizing life phenomena. For the EKIW distribution, the hazard rate function is

$$
\begin{aligned}
h(x ; \alpha, \beta, \lambda, \eta, \theta) & =\frac{\beta \lambda \theta \eta \alpha^{\beta} x^{-(\beta+1)} \exp \left[-\lambda\left(\frac{\alpha}{x}\right)^{\beta}\right]\left(1-\exp \left[-\lambda\left(\frac{\alpha}{x}\right)^{\beta}\right]\right)^{\eta-1}}{1-\left[1-\left(1-\exp \left[-\lambda\left(\frac{\alpha}{x}\right)^{\beta}\right]\right)^{\eta}\right]^{\theta}} \\
& \times\left[1-\left(1-\exp \left[-\lambda\left(\frac{\alpha}{x}\right)^{\beta}\right]\right)^{\eta}\right]^{\theta-1}
\end{aligned}
$$

Figure 2 illustrates some of possible shapes of the hazard function of the EKIW distribution for selected values of the parameters $\alpha, \beta, \lambda, \eta$ and $\theta$.

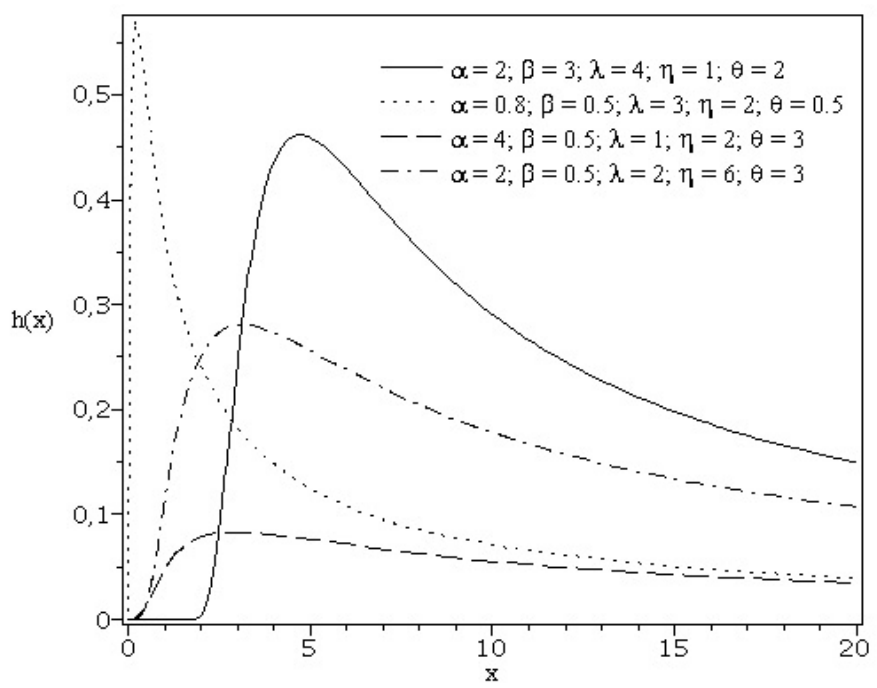

Fig. 2. The hazard function of the EKIW distribution for different values of the parameters.

The reverse hazard function of the EKIW distribution is

$$
\begin{aligned}
h(x ; \alpha, \beta, \lambda, \eta, \theta) & =\frac{\beta \lambda \theta \eta \alpha^{\beta} x^{-(\beta+1)} \exp \left[-\lambda\left(\frac{\alpha}{x}\right)^{\beta}\right]\left(1-\exp \left[-\lambda\left(\frac{\alpha}{x}\right)^{\beta}\right]\right)^{\eta-1}}{\left[1-\left(1-\exp \left[-\lambda\left(\frac{\alpha}{x}\right)^{\beta}\right]\right)^{\eta}\right]^{\theta}} \\
& \times\left[1-\left(1-\exp \left[-\lambda\left(\frac{\alpha}{x}\right)^{\beta}\right]\right)^{\eta}\right]^{\theta-1}
\end{aligned}
$$




\subsection{Moments}

Many of the interesting characteristics and features of a distribution can be studied through its moments (e.g. tendency, dispersion, skewness and kurtosis). Therefore, it is customary to derive the moments when a new distribution is proposed.

Using the form in (3.5), we can write

$$
\begin{aligned}
E\left(X^{n}\right) & =\int_{0}^{\infty} x^{n} f(x ; \alpha, \beta, \lambda, \eta, \theta) d x \\
& =\int_{0}^{\infty} \frac{\beta \lambda \theta \eta \alpha^{\beta}}{x^{\beta-n+1}} \sum_{j=0}^{\infty} \sum_{k=0}^{\infty} \frac{(-1)^{j+k} \Gamma(\theta) \Gamma[\eta(j+1)]}{\Gamma(\theta-j) \Gamma[\eta(j+1)-k] j ! k !} \exp \left[-\lambda(k+1)\left(\frac{\alpha}{x}\right)^{\beta}\right] d x \\
& =\beta \lambda \theta \eta \sum_{j=0}^{\infty} \sum_{k=0}^{\infty} \frac{\alpha^{\beta}(-1)^{j+k} \Gamma(\theta) \Gamma[\eta(j+1)]}{\Gamma(\theta-j) \Gamma[\eta(j+1)-k] j ! k !} \int_{0}^{\infty} x^{-(\beta-n+1)} \exp \left[-\lambda(k+1)\left(\frac{\alpha}{x}\right)^{\beta}\right] d x
\end{aligned}
$$

Making the transformation $t=\lambda(k+1)(\alpha / x)^{\beta}$ and using the definition of the gamma function (3.2), the $n$th moment of EKIW distribution is given by

$$
E\left(X^{n}\right)=\lambda \theta \eta \alpha^{n} \sum_{j=0}^{\infty} \sum_{k=0}^{\infty} \frac{(-1)^{j+k} \Gamma(\theta) \Gamma[\eta(j+1)] \Gamma\left(1-\frac{n}{\beta}\right)}{[\lambda(k+1)]^{1-n / \beta} \Gamma(\theta-j) \Gamma[\eta(j+1)-k] j ! k !}
$$

for $n<\beta$. In particular, the mean for the EKIW distribution is given by

$$
\mu=E(X)=\lambda \theta \eta \alpha \sum_{j=0}^{\infty} \sum_{k=0}^{\infty} \frac{(-1)^{j+k} \Gamma(\theta) \Gamma[\eta(j+1)] \Gamma\left(1-\frac{1}{\beta}\right)}{[\lambda(k+1)]^{1-1 / \beta} \Gamma(\theta-j) \Gamma[\eta(j+1)-k] j ! k !}
$$

Table 1 contains the mean of the EKIW distribution for various values of parameters $\alpha, \beta, \lambda, \eta$ and $\theta$.

Table 1. Mean of the EKIW distribution for different values of the parameters.

\begin{tabular}{cccccc}
\hline$\alpha$ & $\beta$ & $\lambda$ & $\eta$ & $\theta$ & Mean \\
\hline 0.2 & 1.5 & 1.0 & 1.0 & 1.0 & 0.536 \\
0.5 & 1.5 & 1.0 & 1.0 & 1.0 & 1.339 \\
1.0 & 1.5 & 1.0 & 1.0 & 1.0 & 2.679 \\
1.0 & 2.0 & 1.0 & 1.0 & 1.0 & 1.772 \\
1.0 & 2.0 & 1.5 & 1.0 & 1.0 & 2.171 \\
1.0 & 2.0 & 2.0 & 1.0 & 1.0 & 2.507 \\
1.0 & 2.0 & 2.0 & 1.0 & 1.5 & 3.760 \\
1.5 & 3.5 & 2.5 & 1.0 & 2.5 & 2.575 \\
2.0 & 1.5 & 2.0 & 3.0 & 4.0 & 4.216 \\
3.0 & 2.0 & 2.0 & 1.0 & 2.5 & 10.540 \\
3.0 & 4.0 & 2.0 & 4.0 & 1.5 & 4.687 \\
3.0 & 5.5 & 6.0 & 3.0 & 6.5 & 3.848 \\
4.0 & 2.0 & 2.0 & 4.0 & 3.5 & 6.814 \\
7.0 & 5.0 & 6.0 & 4.0 & 6.5 & 8.801 \\
\hline
\end{tabular}




\section{J. A. Rodrigues et al.}

\subsection{Quantile function}

The quantile function $Q(p), 0<p<1$, for the EKIW distribution is given by

$$
Q(p)=\frac{\alpha}{\left\{-\log \left[1-\left(1-p^{1 / \theta}\right)^{1 / \eta}\right]^{1 / \lambda}\right\}^{1 / \beta}}
$$

The median of the distribution is obtained by using $p=0.5$ in (3.11). The random sample can also be easily generated from (3.11) by using $p$ as uniform random number.

\subsection{Mean deviations}

The amount of spread in a population is evidently measured to some extent by the totality of deviations from the mean and median. These are known as the mean deviation about the mean and the mean deviation about the median. Let $X$ be a EKIW random variable with mean $\mu=E(X)$ and median $m$.

The mean deviation from the mean can be defined as

$$
\begin{aligned}
\delta_{1}(X) & =E(|X-\mu|) \\
& =\int_{0}^{\infty}|x-\mu| f(x ; \alpha, \beta, \lambda, \eta, \theta) d x \\
& =2 \mu F(\mu ; \alpha, \beta, \lambda, \eta, \theta)-2 \mu+2 \int_{\mu}^{\infty} x f(x ; \alpha, \beta, \lambda, \eta, \theta) d x \\
& =2 \mu\left[1-\left(1-\exp \left[-\lambda\left(\frac{\alpha}{\mu}\right)^{\beta}\right]\right)^{\eta}\right]^{\theta}-2 \mu \\
& +2 \lambda \theta \eta \alpha \sum_{j=0}^{\infty} \sum_{k=0}^{\infty} \frac{(-1)^{j+k} \Gamma(\theta) \Gamma[\eta(j+1)][\lambda(k+1)]^{-1+1 / \beta}}{\Gamma(\theta-j) \Gamma[\eta(j+1)-k] j ! k !} \\
& \times \gamma\left(1-\frac{1}{\beta}, \frac{\lambda(k+1) \alpha^{\beta}}{\mu^{\beta}}\right)
\end{aligned}
$$

where $\gamma(\cdot, \cdot)$ is the lower incomplete gamma function defined by

$$
\gamma(\alpha, x)=\int_{0}^{x} t^{\alpha-1} e^{-t} d t
$$

The mean deviation from the median is, also, defined by

$$
\begin{aligned}
\delta_{1}(X) & =E(|X-m|) \\
& =\int_{0}^{\infty}|x-m| f(x ; \alpha, \beta, \lambda, \eta, \theta) d x \\
& =-\mu+2 \int_{m}^{\infty} x f(x ; \alpha, \beta, \lambda, \eta, \theta) d x \\
& =-\mu+2 \lambda \theta \eta \alpha \sum_{j=0}^{\infty} \sum_{k=0}^{\infty} \frac{(-1)^{j+k} \Gamma(\theta) \Gamma[\eta(j+1)][\lambda(k+1)]^{-1+1 / \beta}}{\Gamma(\theta-j) \Gamma[\eta(j+1)-k] j ! k !} \\
& \times \gamma\left(1-\frac{1}{\beta}, \frac{\lambda(k+1) \alpha^{\beta}}{m^{\beta}}\right)
\end{aligned}
$$




\subsection{Bonferroni and Lorenz curves}

The Bonferroni and Lorenz curves have applications not only in economics to study income and poverty, but also in other fields like reliability, demography, insurance and medicine. The Bonferroni and Lorenz curves are defined by

$$
B(p)=\frac{1}{p \mu} \int_{0}^{q} x f(x) d x
$$

and

$$
L(p)=\frac{1}{\mu} \int_{0}^{q} x f(x) d x
$$

respectively, where $\mu=E(X)$ and $q=F^{-1}(p)$. In the case of EKIW distribution, we obtain

$$
B(p)=\frac{\lambda \theta \eta \alpha}{p \mu} \sum_{j=0}^{\infty} \sum_{k=0}^{\infty} \frac{(-1)^{j+k} \Gamma(\theta) \Gamma[\eta(j+1)][\lambda(j+1)]^{-1+1 / \beta}}{\Gamma(\theta-j) \Gamma[\eta(j+1)-k] j ! k !} \Gamma\left[1-\frac{1}{\beta}, \frac{\lambda(j+1) \alpha^{\beta}}{q^{\beta}}\right]
$$

and

$$
L(p)=\frac{\lambda \theta \eta \alpha}{\mu} \sum_{j=0}^{\infty} \sum_{k=0}^{\infty} \frac{(-1)^{j+k} \Gamma(\theta) \Gamma[\eta(j+1)][\lambda(j+1)]^{-1+1 / \beta}}{\Gamma(\theta-j) \Gamma[\eta(j+1)-k] j ! k !} \Gamma\left[1-\frac{1}{\beta}, \frac{\lambda(j+1) \alpha^{\beta}}{q^{\beta}}\right]
$$

where $\Gamma(\cdot, \cdot)$ is the upper incomplete gamma function defined by

$$
\Gamma(\alpha, x)=\int_{x}^{\infty} t^{\alpha-1} e^{-t} d t
$$

\subsection{Entropy}

An entropy of a random variable $X$ is a measure of variation of the uncertainty. It is an important concept in many fields of science, especially theory of communication, physics and probability. A popular entropy measure is Rényi entropy. If $X$ has the PDF $f(\cdot)$ then Rényi entropy is defined by

$$
H_{R}(v)=\frac{1}{1-v} \log \left[\int f^{v}(x) d x\right]
$$

where $v>0$ and $v \neq 1$. Using (3.20), Rényi entropy of EKIW distribution is given by

$$
\begin{aligned}
& H_{R}(v)=\frac{v}{1-v}(\log \beta+\log \lambda+\log \theta+\log \eta+\beta \log \alpha)+\frac{1-v(\beta+1)}{1-v} \log \alpha-\log \beta \\
& +\frac{1}{1-v} \log \sum_{j=0}^{\infty} \sum_{k=0}^{\infty} \frac{(-1)^{j+k} \Gamma[v(\theta-1)+1] \Gamma[\eta j+v(\eta-1)+1][\lambda(\nu+k)]^{[1-v(\beta+1)] / \beta}}{\Gamma[v(\theta-1)+1-j] \Gamma[j \eta+v(\eta-1)+1-k] j ! k !} \\
& \times \Gamma\left[\frac{v(\beta+1)-1}{\beta}\right]
\end{aligned}
$$




\section{J. A. Rodrigues et al.}

\subsection{Order statistics}

Order statistics are among the most fundamental tools in non-parametric statistics and inference. They enter in the problems of estimation and hypothesis tests in a variety of ways. Therefore, we now discuss some properties of the order statistics for the EKIW distribution. Suppose $X_{1}, X_{2}, \ldots, X_{n}$ is a random sample from EKIW distribution. Let $X_{1: n}<X_{2: n}<\ldots<X_{n: n}$ denote the corresponding order statistics. From [1], the PDF and CDF of the $r$ th order statistic, say $Y=X_{r: n}$, are given by

$$
\begin{aligned}
f_{Y}(y) & =\frac{n !}{(r-1) !(n-r) !} F^{r-1}(y)[1-F(y)]^{n-r} f(y) \\
& =\frac{n !}{(r-1) !(n-r) !} \sum_{l=0}^{n-r}\left(\begin{array}{c}
n-r \\
l
\end{array}\right)(-1)^{l} F^{l+r-1}(y) f(y)
\end{aligned}
$$

and

$$
\begin{aligned}
F_{Y}(y) & =\sum_{j=r}^{n}\left(\begin{array}{c}
n \\
j
\end{array}\right) F^{j}(y)[1-F(y)]^{n-j} \\
& =\sum_{j=r}^{n} \sum_{l=0}^{n-j}\left(\begin{array}{c}
n \\
j
\end{array}\right)\left(\begin{array}{c}
n-j \\
l
\end{array}\right)(-1)^{l} F^{j+l}(y)
\end{aligned}
$$

where $f(\cdot)$ and $F(\cdot)$ are the PDF and CDF of the EKIW distribution, respectively. It follows from Equations (2.7) and (2.8) that

$$
\begin{aligned}
f_{Y}(y) & =\frac{\beta \lambda \theta \eta \alpha^{\beta} y^{-(\beta+1)} n !}{(r-1) !(n-r) !} \exp \left[-\lambda\left(\frac{\alpha}{y}\right)^{\beta}\right]\left(1-\exp \left[-\lambda\left(\frac{\alpha}{y}\right)^{\beta}\right]\right)^{\eta-1} \\
& \times \sum_{l=0}^{n-r}\left(\begin{array}{c}
n-r \\
l
\end{array}\right)(-1)^{l}\left[1-\left(1-\exp \left[-\lambda\left(\frac{\alpha}{y}\right)^{\beta}\right]\right)^{\eta}\right]^{\theta(l+r)-1}
\end{aligned}
$$

and

$$
F_{Y}(y)=\sum_{j=r}^{n} \sum_{l=0}^{n-j}\left(\begin{array}{c}
n \\
j
\end{array}\right)\left(\begin{array}{c}
n-j \\
l
\end{array}\right)(-1)^{l}\left[1-\left(1-\exp \left[-\lambda\left(\frac{\alpha}{y}\right)^{\beta}\right]\right)^{\eta}\right]^{\theta(j+l)}
$$

\section{Characterizations of model}

Characterizations of distributions are important to many researchers in the applied fields. An investigator will be vitally interested to know if their model fits the requirements of a particular distribution. To this end, one will depend on the characterizations of this distribution which provide conditions under which the underlying distribution is indeed that particular distribution. Various characterizations of distributions have been established in many different directions. In this section, several characterizations of (EKIW) distribution are presented. These characterizations are based on: $(i)$ a simple relationship between two truncated moments; (ii) a single function of the random variable.

\subsection{Characterizations based on truncated moments}

In this subsection we present characterizations of (EKIW) distribution in terms of a simple relationship between two truncated moments. Our characterization results presented here will employ an 
interesting result due to [3] (Theorem G, below). The advantage of the characterizations given here is that, $c d f F$ need not have a closed form and are given in terms of an integral whose integrand depends on the solution of a first order differential equation, which can serve as a bridge between probability and differential equation.

Theorem G. Let $(\Omega, \mathscr{F}, \mathbf{P})$ be a given probability space and let $H=[a, b]$ be an interval for some $a<b \quad(a=-\infty, b=\infty$ might as well be allowed $)$. Let $X: \Omega \rightarrow H$ be a continuous random variable with the distribution function $F$ and let $g$ and $h$ be two real functions defined on $H$ such that

$$
\mathbf{E}[g(X) \mid X \geq x]=\mathbf{E}[h(X) \mid X \geq x] \zeta(x), \quad x \in H,
$$

is defined with some real function $\zeta$. Assume that $g, h \in C^{1}(H), \zeta \in C^{2}(H)$ and $F$ is twice continuously differentiable and strictly monotone function on the set $H$. Finally, assume that the equation $h \zeta=g$ has no real solution in the interior of $H$. Then $F$ is uniquely determined by the functions $g, h$ and $\zeta$, particularly

$$
F(x)=\int_{a}^{x} C\left|\frac{\zeta^{\prime}(u)}{\zeta(u) h(u)-g(u)}\right| \exp (-s(u)) d u
$$

where the function $s$ is a solution of the differential equation $s^{\prime}=\frac{\zeta^{\prime} h}{\zeta h-g}$ and $C$ is a constant, chosen to make $\int_{H} d F=1$.

Clearly, Theorem $\mathrm{G}$ can be stated in terms of two functions $g$ and $\zeta$ by taking $h(x) \equiv 1$, which will reduce the condition given in Theorem $\mathrm{G}$ to $E[g(X) \mid X \geq x]=\zeta(x)$. However, adding an extra function will give a lot more flexibility, as far as its application is concerned.

Proposition 4.1. Let $X: \Omega \rightarrow(0, \infty)$ be a continuous random variable and let $h(x)=$ $\left[1-\left(1-e^{-\lambda\left(\frac{\alpha}{x}\right)^{\beta}}\right)^{\eta}\right]^{1-\theta}$ and $g(x)=h(x)\left(1-e^{-\lambda\left(\frac{\alpha}{x}\right)^{\beta}}\right)$ for $x \in(0, \infty)$. The pdf of $X$ is (2.8) if and only if the function $\zeta$ defined in Theorem $\mathrm{G}$ has the form

$$
\zeta(x)=\frac{a}{a+1}\left(1-e^{-\lambda\left(\frac{\alpha}{x}\right)^{\beta}}\right), \quad x>0 .
$$

Proof. Let $X$ have density $(2.8)$, then 


$$
(1-F(x)) \mathbf{E}[h(X) \mid X \geq x]=b\left(1-e^{-\lambda\left(\frac{\alpha}{x}\right)^{\beta}}\right)^{\eta}, \quad x>0
$$

and

$$
(1-F(x)) \mathbf{E}[g(X) \mid X \geq x]=\frac{a b}{a+1}\left(1-e^{-\lambda\left(\frac{\alpha}{x}\right)^{\beta}}\right)^{\eta+1}, \quad x>0
$$

and finally

$$
\zeta(x) h(x)-g(x)=-\frac{1}{a+1} h(x)\left(1-e^{-\lambda\left(\frac{\alpha}{x}\right)^{\beta}}\right)<0 \text { for } x>0 .
$$

Conversely, if $\zeta$ is given as above, then

$$
s^{\prime}(x)=\frac{\zeta^{\prime}(x) h(x)}{\zeta(x) h(x)-g(x)}=\frac{\beta \lambda \eta \alpha^{\beta} x^{-(\beta+1)} e^{-\lambda\left(\frac{\alpha}{x}\right)^{\beta}}}{1-e^{-\lambda\left(\frac{\alpha}{x}\right)^{\beta}}}, x>0
$$

and hence

$$
s(x)=-\ln \left\{\left(1-e^{-\lambda\left(\frac{\alpha}{x}\right)^{\beta}}\right)^{\eta}\right\}, \quad x>0 .
$$

Now, in view of Theorem G, $X$ has density (2.8).

Corollary 4.1. Let $X: \Omega \rightarrow(0, \infty)$ be a continuous random variable and let $h(x)$ be as in

Proposition 1. The $p d f$ of $X$ is (2.8) if and only if there exist functions $g$ and $\zeta$ defined in Theorem $\mathrm{G}$ satisfying the differential equation

$$
\frac{\zeta^{\prime}(x) h(x)}{\zeta(x) h(x)-g(x)}=\frac{\beta \lambda \eta \alpha^{\beta} x^{-(\beta+1)} e^{-\lambda\left(\frac{\alpha}{x}\right)^{\beta}}}{1-e^{-\lambda\left(\frac{\alpha}{x}\right)^{\beta}}}, \quad x>0 .
$$

Remarks 4.1. (a) The general solution of the differential equation in Corollary 4.1 is

$$
\zeta(x)=\left(1-e^{-\lambda\left(\frac{\alpha}{x}\right)^{\beta}}\right)^{-\eta}\left[-\int \beta \lambda \eta \alpha^{\beta} x^{-(\beta+1)} e^{-\lambda\left(\frac{\alpha}{x}\right)^{\beta}}\left(1-e^{-\lambda\left(\frac{\alpha}{x}\right)^{\beta}}\right)^{\eta-1}(h(x))^{-1} g(x) d x+D\right],
$$

for $x>0$, where $D$ is a constant. One set of appropriate functions is given in Proposition A.1 with $D=0$.

(b) Clearly there are other triplets of functions $(h, g, \zeta)$ satisfying the conditions of Theorem G. We presented one such triplet in Proposition 4.1. 


\subsection{Characterizations based on single function of the random variable}

In this subsection we employ a single function $\psi$ of $X$ and state characterization results in terms of $\psi(X)$. The following proposition has already appeared a Technical Report [6], so we will just state it here for the sake of completeness.

Proposition 4.2 Let $X: \Omega \rightarrow(a, b)$ be a continuous random variable with $c d f F$. Let $\psi(x)$ be a differentiable function on $(a, b)$ with $\lim _{x \rightarrow b} \psi(x)=1$. Then for $\delta \neq 1$,

$$
E[\psi(X) \mid X<x]=\delta \psi_{1}(x), \quad x \in(a, b)
$$

if and only if

$$
\psi(x)=(F(x))^{\frac{1}{\delta}-1}, \quad x \in(a, b)
$$

Remark 4.2. Taking, e.g., $\psi(x)=\left\{\left[1-\left(1-e^{-\lambda\left(\frac{\alpha}{x}\right)^{\beta}}\right)^{\eta}\right]\right\}^{\frac{b \delta}{1-\delta}}$, Proposition 4.2 gives a characterization of $(2.8)$.

\section{Estimation of model parameters}

In this section, we consider estimation of the five parameters by the methods of moments and maximum likelihood. Suppose $x_{1}, \ldots, x_{n}$ is a random sample of size $n$ from the EKIW distribution given by (2.7). Under the method of moments, equating the theoretical moments $E\left(X^{r}\right)$ with the corresponding sample moments,

$$
M_{r}=\frac{1}{n} \sum_{l=1}^{n} x_{l}^{r}, r=1, \ldots, 5
$$

respectively, one obtains the system of equations

$$
M_{r}=\lambda \theta \eta \alpha^{r} \sum_{j=0}^{\infty} \sum_{k=0}^{\infty} \frac{(-1)^{j+k} \Gamma(\theta) \Gamma[\eta(j+1)] \Gamma\left(1-\frac{r}{\beta}\right)}{[\lambda(k+1)]^{1-r / \beta} \Gamma(\theta-j) \Gamma[\eta(j+1)-k] j ! k !}
$$

which can be solved simultaneously to give estimates for $\alpha, \beta, \lambda, \eta$ and $\theta$. 


\section{J. A. Rodrigues et al.}

Now consider estimation by the method of maximum likelihood. The log-likelihood for a random sample $x_{1}, \ldots, x_{n}$ from the EKIW distribution given by (2.7) is

$$
\begin{aligned}
\log L(\alpha, \beta, \lambda, \eta, \theta) & =n \log \beta+n \log \lambda+n \log \theta+n \log \eta+n \beta \log \alpha-(\beta+1) \sum_{i=1}^{n} \log x_{i} \\
& -\lambda \sum_{i=1}^{n}\left(\frac{\alpha}{x_{i}}\right)^{\beta}+(\eta-1) \sum_{i=1}^{n} \log \left(1-\exp \left[-\lambda\left(\frac{\alpha}{x_{i}}\right)^{\beta}\right]\right) \\
& +(\theta-1) \sum_{i=1}^{n} \log \left[1-\left(1-\exp \left[-\lambda\left(\frac{\alpha}{x_{i}}\right)^{\beta}\right]\right)^{\eta}\right]
\end{aligned}
$$

Differentiating the $\log$-likelihood with respect $\alpha, \beta, \lambda, \eta$ and $\theta$, respectively, and setting the result equal to zero, we have

$$
\begin{aligned}
& \frac{\partial \log L}{\partial \alpha}=\frac{\beta \lambda \eta(1-\theta)}{\alpha} \sum_{i=1}^{n} \frac{\left(\frac{\alpha}{x_{i}}\right)^{\beta} \exp \left[-\lambda\left(\frac{\alpha}{x_{i}}\right)^{\beta}\right]\left(1-\exp \left[-\lambda\left(\frac{\alpha}{x_{i}}\right)^{\beta}\right]\right)^{\eta-1}}{1-\left(1-\exp \left[-\lambda\left(\frac{\alpha}{x_{i}}\right)^{\beta}\right]\right)^{\eta}} \\
& +\frac{n \beta}{\alpha}-\frac{\lambda}{\alpha} \sum_{i=1}^{n}\left(\frac{\alpha}{x_{i}}\right)^{\beta}-\beta \lambda(\eta-1) \alpha^{\beta-1} \sum_{i=1}^{n} \frac{x_{i}^{-\beta}}{1-\exp \left[\lambda\left(\frac{\alpha}{x_{i}}\right)^{\beta}\right]}=0 \\
& \begin{aligned}
\frac{\partial \log L}{\partial \beta}=\frac{n}{\beta}+n \log \alpha-\sum_{i=1}^{n} \log x_{i}+\lambda \sum_{i=1}^{n}\left(\frac{\alpha}{x_{i}}\right)^{\beta} \log \left(\frac{\alpha}{x_{i}}\right)+(1-\eta) \sum_{i=1}^{n} \frac{\lambda\left(\frac{\alpha}{x_{i}}\right)^{\beta} \log \left(\frac{\alpha}{x_{i}}\right)}{1-\exp \left[\lambda\left(\frac{\alpha}{x_{i}}\right)^{\beta}\right]} \\
-\lambda \eta(\theta-1) \sum_{i=1}^{n} \frac{\left(\frac{\alpha}{x_{i}}\right)^{\beta} \log \left(\frac{\alpha}{x_{i}}\right) \exp \left[-\lambda\left(\frac{\alpha}{x_{i}}\right)^{\beta}\right]\left(1-\exp \left[-\lambda\left(\frac{\alpha}{x_{i}}\right)^{\beta}\right]\right)^{\eta-1}}{1-\left(1-\exp \left[-\lambda\left(\frac{\alpha}{x_{i}}\right)^{\beta}\right]\right)^{\eta}}=0
\end{aligned} \\
& \frac{\partial \log L}{\partial \lambda}=\eta(1-\theta) \sum_{i=1}^{n} \frac{\left(\frac{\alpha}{x_{i}}\right)^{\beta} \exp \left[-\lambda\left(\frac{\alpha}{x_{i}}\right)^{\beta}\right]\left(1-\exp \left[-\lambda\left(\frac{\alpha}{x_{i}}\right)^{\beta}\right]\right)^{\eta-1}}{1-\left(1-\exp \left[-\lambda\left(\frac{\alpha}{x_{i}}\right)^{\beta}\right]\right)^{\eta}} \\
& +\frac{n}{\lambda}+\sum_{i=1}^{n}\left(\frac{\alpha}{x_{i}}\right)^{\beta}-(\eta-1) \alpha^{\beta} \sum_{i=1}^{n} \frac{x_{i}^{-\beta}}{1-\exp \left[\lambda\left(\frac{\alpha}{x_{i}}\right)^{\beta}\right]}=0
\end{aligned}
$$




$$
\begin{aligned}
\frac{\partial \log L}{\partial \eta} & =\frac{n}{\eta}+(\theta-1) \sum_{i=1}^{n} \frac{\log \left(1-\exp \left[-\lambda\left(\frac{\alpha}{x_{i}}\right)^{\beta}\right]\right)}{1-\left(1-\exp \left[-\lambda\left(\frac{\alpha}{x_{i}}\right)^{\beta}\right]\right)^{-\eta}} \\
& +\sum_{i=1}^{n} \log \left(1-\exp \left[-\lambda\left(\frac{\alpha}{x_{i}}\right)^{\beta}\right]\right)=0 \\
\frac{\partial \log L}{\partial \theta} & =\frac{n}{\theta}+\sum_{i=1}^{n} \log \left[1-\left(1-\exp \left[-\lambda\left(\frac{\alpha}{x_{i}}\right)^{\beta}\right]\right)^{\eta}\right]=0
\end{aligned}
$$

The maximum likelihood estimates $\widehat{\alpha}, \widehat{\beta}, \widehat{\lambda}, \widehat{\eta}$ and $\widehat{\theta}$ of the unknown parameters $\alpha, \beta, \lambda, \eta$ and $\theta$, respectively, are obtained by solving Equations 5.4-5.8.

\section{Application}

The data set given in Table 2 represents the relief times of twenty patients receiving an analgesic. This data set was taken from [4]. We would like to emphasize that the aim here is not to provide a complete statistical modeling or inferences for the data set involved.

Table 2. Relief times of twenty patients.

\begin{tabular}{llllllllll}
\hline 1.1 & 1.4 & 1.3 & 1.7 & 1.9 & 1.8 & 1.6 & 2.2 & 1.7 & 2.7 \\
4.1 & 1.8 & 1.5 & 1.2 & 1.4 & 3.0 & 1.7 & 2.3 & 1.6 & 2.0 \\
\hline
\end{tabular}

We use the lifetime data set given by Table 2 to compare the fit of the EKIW distribution with four distributions: gamma, IW, Lindley and Weibull. The PDF of gamma, IW, Lindley and Weibull distributions are

(1) Gamma:

$$
f(x)=\frac{\beta^{\alpha} x^{\alpha-1}}{\Gamma(\alpha)} \exp (-\beta x)
$$

(2) IW:

$$
f(x)=\beta \alpha^{\beta} x^{-(\beta+1)} \exp \left[-\left(\frac{\alpha}{x}\right)^{\beta}\right]
$$

(3) Lindley:

$$
f(x)=\frac{\alpha^{2}}{\alpha+1}(1+x) \exp (-\alpha x)
$$

(4) Weibull:

$$
f(x)=\alpha \beta(\beta x)^{\alpha-1} \exp \left[-(\beta x)^{\alpha}\right]
$$




\section{J. A. Rodrigues et al.}

The maximum likelihood estimates and the Akaike Information Criterion (AIC) values for the fitted distributions are reported in Table 3. The results show that the EKIW distribution provides a significantly better fit than the other four models.

Table 3. The maximum likelihood estimates and AIC of the models based on data set.

\begin{tabular}{llc}
\hline Distribution & \multicolumn{1}{c}{ Maximum Likelihood Estimates } & AIC \\
\hline EKIW & $\alpha=4.324, \beta=2.319, \lambda=0.567, \eta=3.166, \theta=0.228$ & 32.509 \\
Gamma & $\alpha=9.670, \beta=5.089$ & 39.637 \\
IW & $\alpha=1.563, \beta=4.017$ & 34.817 \\
Lindley & $\alpha=0.816$ & 62.499 \\
Weibull & $\alpha=2.130, \beta=2.787$ & 45.173 \\
\hline
\end{tabular}

Plots of the estimated PDF of the EKIW, gamma, IW, Lindley and Weibull models fitted to these data set are given in Figure 3. The figure indicate that the EKIW distribution is superior to the other distributions in terms of model fitting.

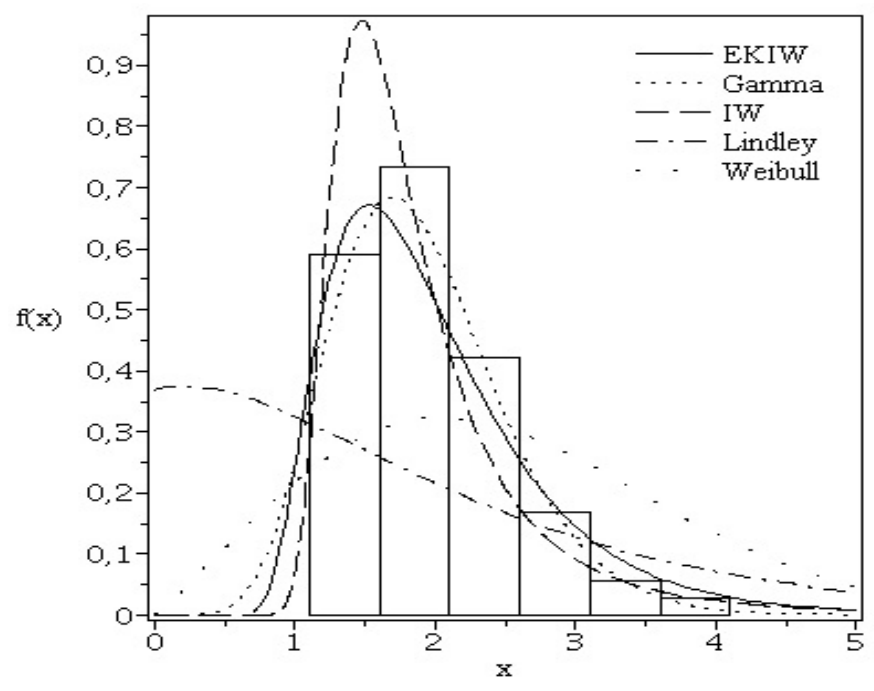

Fig. 3. Histogram and estimated densities.

\section{Conclusion}

We proposed a new distribution, named the exponentiated Kumaraswamy inverse Weibull distribution which extends the inverse Weibull distribution. Several properties of the new distribution were investigated, including the moments, hazard function, mean deviations and Rényi entropy. The estimation of parameters by the method of moments and the maximum likelihood have been discussed. An application of the exponentiated Kumaraswamy inverse Weibull distribution to real data show that the new distribution can be used quite effectively to provide better fits than the gamma, inverse Weibull, Lindley and Weibull distributions. 


\section{References}

[1] B.C. Arnold, A.N. Balakrishnan, and H.N. Nagaraja, A first course in order statistics, WileyInterscience, New York, 1992.

[2] L.A. Baharith, S.A. Mousa, M.A. Atallah and S.H. Elgayar, The beta generalized inverse Weibull distribution, British Journal of Mathematics and Computer Science. 4 (2014), pp. 252-270.

[3] W. Glänzel, A characterization theorem based on truncated moments and its application to some distribution families, Mathematical Statistics and Probability Theory (Bad Tatzmannsdorf, 1986), Vol. B, Reidel, Dordrecht, 1987, pp. 75-84.

[4] A.J. Gross and V.A. Clark, Survival distributions: Reliability applications in the biomedical sciences, Wiley-Interscience, New York, 1975.

[5] F.R.S. Gusmão, E.M.M. Ortega and G.M. Cordeiro, The generalized inverse Weibull distribution, Statistical Papers. 52 (2011), pp. 591-619.

[6] G.G. Hamedani, On certain generalized gamma convolution distributions II, Technical Report, No. 484, MSCS, Marquette University (2013).

[7] S. Huang and B.O. Oluyede, Exponentiated Kumaraswamy-Dagum distribution with applications to income and lifetime data, Journal of Statistical Distributions and Applications. 1 (8) (2014), pp. 1-18.

[8] A.Z. Keller and A.R. Kamath, Reliability analysis of CNC Machine Tools, Reliability Engineering. 3 (6) (1982), pp. 449-473.

[9] M.S. Khan and R. King, Modified inverse Weibull distribution, Journal of Statistics Applications and Probability. 1(2) (2012), pp. 115-132.

[10] M.S. Khan, The beta inverse Weibull distribution, International Transactions in Mathematical Sciences and Computer. 3 (2010), pp. 113-119,

[11] P. Kumaraswamy, A generalized probability density function for double-bounded random processes, Journal of Hydrology. 46 (1980), pp. 79-88.

[12] A.J. Lemonte, W.B. Souza and G.M. Cordeiro, The exponentiated Kumaraswamy distribution and its log-transform, Brazilian Journal of Probability and Statistics. 27(1) (2013), pp. 31-53.

[13] M.Q. Shahbaz, S. Shahbaz and N.S. Butt, The KumaraswamyInverse Weibull Distribution, Statistics in the Twenty-First Century. 8 (2012), pp. 479-489.

[14] V.G. Vodã, On the Inverse Rayleigh Distribution Random Variable, Rep Statistical Applied Research JUSE. 19 (1972), pp. 13-21. 